\title{
A Review of Clinical and Laboratory Predictors of Severe COVID-19 Disease
}

\author{
Edward Kwon, MD, Nathan Whitlow, MD, and Alexander Reed, MD
}

Introduction: In late December 2019, the coronavirus 2 (SARS-CoV-2) emerged in Wuhan, China. It quickly spread and emerged as a global pandemic with far-reaching impacts on society. As clinical research on this novel virus emerges, there is a limited amount of data that review clinical and laboratory predictors of severe disease. We present a case of a patient with severely elevated inflammatory markers who remained clinically stable during his hospital course.

Case discussion: A 53-year-old male presented to the emergency room with 11 days of persistent fevers and new-onset anterior chest tightness. He was admitted to the hospital due to a reported oxygen desaturation at home to $87 \%$ (taken by his spouse, a healthcare professional) and ambulatory oxygen desaturation down to $87 \%$. He was noted to have severely elevated inflammatory markers,

lymphopenia, and computed tomography pulmonary angiograph findings consistent with COVID-19. He remained on room air and clinically stable throughout his 3-day hospital course. While his C-reactive protein levels improved, his ferritin and erythrocyte sedimentation rate continued to elevate. He was discharged home and was symptom-free within 4 days of hospital discharge.

Discussion: COVID-19 has proven to be a viral disease with a high transmission rate, that has caused over 100,000 deaths in the United States, thus far. The decision to admit a patient must balance the risks of transmission with the benefit of being readily available to provide urgent supportive care should the patient develop complications. Thus, there is a significant benefit to being able to predict poor outcomes. We performed a targeted review of the literature, focusing on clinical and laboratory predictors of poor outcomes in COVID-19. Our case report and narrative review outline these findings within the context of our case. ( $\mathrm{J}$ Am Board Fam Med 2021;34:S186-S191.)

Keywords: Blood Sedimentation, Communicable Diseases, CoVID-19, C-reactive protein, Erythrocyte sedimentation rate, Ferritins, Fever, Lymphopenia, Pandemics

\section{Introduction}

In late December 2019, a pneumonia caused by the novel severe acute respiratory syndrome coronavirus 2 (SARS-CoV-2) emerged in Wuhan, Hubei Province, China. This novel virus spread across the globe resulting in a pandemic and worldwide

This article was externally peer reviewed.

Submitted 22 April 2020; revised 26 June 2020; accepted 29 June 2020.

From the Department of Family Medicine, Fort Belvoir Community Hospital, Fort Belvoir, VA (All authors).

Funding: The authors received no financial support for the research, authorship, and/or publication of this article.

Conflict of interest: None.

Disclosures: The opinions and assertions contained herein are the private views of the authors and are not to be construed as official or as reflecting the views of the Department of the Army, Department of the Air Force, Department of the Navy, Department of Defense, or the U.S. government. This document was created free of branding or market affiliations. The authors are operating solely as contributors. effects. ${ }^{1,2}$ The World Health Organization named the disease caused by SARS-CoV2, Coronavirus disease 2019 (COVID-19). While studies have evaluated clinical and laboratory characteristics of COVID-19 fatalities and patients with severe disease, there is limited data on those with mild disease. This creates challenges predicting which patients will progress to severe disease. This report describes the case of a patient with severely elevated inflammatory markers and moderate lymphopenia, concerning for impending development of severe disease, who did not require supplemental oxygen and remained clinically stable through his hospital course.

Corresponding author: Edward Kwon, MD, 300 Dewitt Loop, Department of Family Medicine, Fort Belvoir, VA 22060 (E-mail: eddiekwon6@yahoo.com). 


\section{Case Report}

A 53-year-old male with a history of hypertension was admitted to the hospital with persistent fevers and a high clinical suspicion for COVID-19. He had no smoking history and was in good aerobic condition. His medication list included Flonase and AmlodipineBenazepril 5-20 mg once daily. The patient had workplace exposure to a known COVID-19 case and subsequently developed subjective fevers, chills, and a mild headache. He presented to the "COVID clinic" on day 3 of symptoms. He was examined, determined to be low-risk, lacked any concerning clinical findings, tested for COVID-19, and dispositioned to home quarantine. His fevers and chills persisted into day 11 of illness. He also developed a decrease in appetite and anterior chest tightness. His wife (a health care professional) applied a home pulse oximeter, which measured oxygen saturation of $87 \%$. The patient returned to the emergency department for evaluation. He endorsed muscle aches and fatigue. He denied any

Figure 1a. Multi-focal, peripheral, rounded ground glass opacities seen on the right upper and adjacent lower lobes (arrows) are commonly reported features of COVID-19.
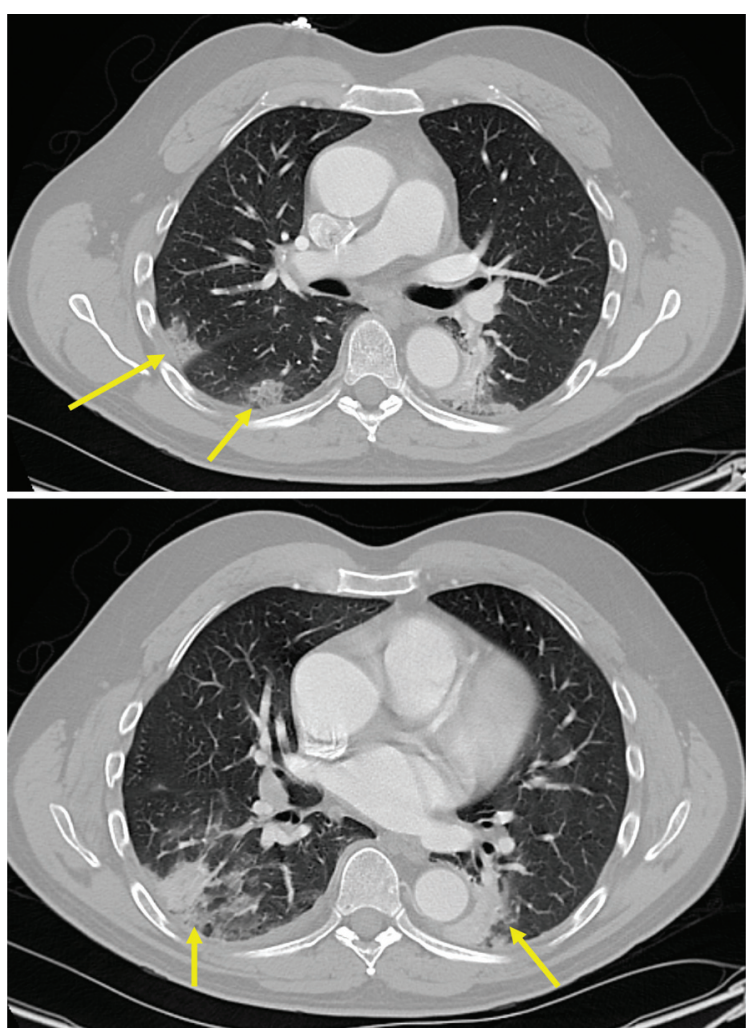

shortness of breath, lightheadedness, nausea, vomiting, or diarrhea.

On examination, his temperature was $99.4^{\circ} \mathrm{F}$, pulse 100 , blood pressure $127 / 78$, respiratory rate 16, and oxygen saturation $97 \%$ on room air. His ambulatory oxygen saturation dropped to $87 \%$.

The patient was awake, alert, appropriate, and in no acute distress. His mucous membranes were moist. His heart examination revealed a regular rate and rhythm without murmurs. His pulmonary examination revealed bibasilar crackles and no wheezing, rales, or rhonchi. His abdominal examination was unremarkable. His extremities were warm and well-perfused.

His laboratory values revealed a white blood cell count of 5.5, a lymphocyte percentage of $15.1 \%$, an absolute lymphocyte count of 0.8 , and a mild neutrophilia. His platelet count was slightly depressed at 127 . His creatine kinase was normal. His highsensitivity troponin was low risk at 6.4 . His inflammatory markers were significant for an erythrocyte sedimentation rate of 55 , C-reactive protein of 8.15 , lactate dehydrogenase of 321 , ferritin of 1698 , and a D-dimer of 0.70. An interleukin-6 level was

Figure 1b. The multi-focal subsegmental consolidations, with ill-defined ground-glass opacities, of the posterior lower lobes (arrows), are nonspecific.
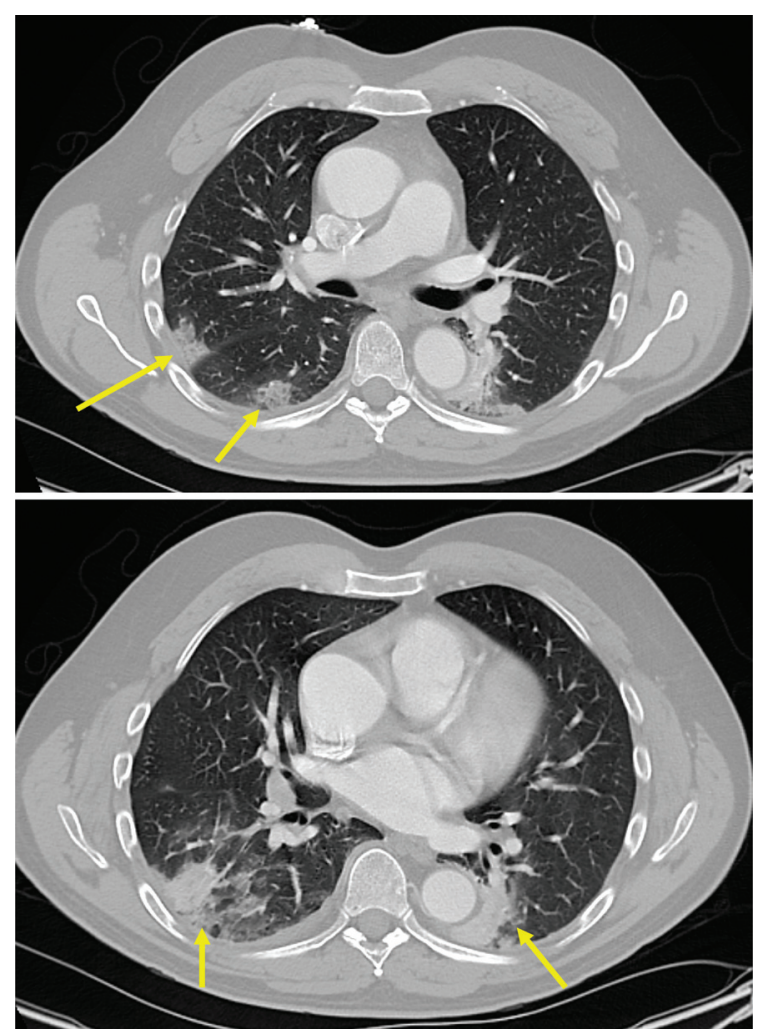
Table 1. Patient Laboratory Values

\begin{tabular}{|c|c|c|c|c|}
\hline Lab & Day 0 & Day 1 & Day 2 & Day 3 \\
\hline $\mathrm{WBC} \times 10^{3} / \mu \mathrm{L}$ & 5.5 & 5.2 & 5.5 & 4.4 \\
\hline Neutrophils abs, $10^{3} / \mu \mathrm{L}$ & 4.1 & 4.1 & 3.8 & 2.6 \\
\hline Neutrophil, \% & 74.4 & 78.1 & 68.7 & 59.3 \\
\hline Lymphocyte abs, $10^{3} / \mu \mathrm{L}$ & 0.8 & 0.6 & 1.0 & 1.1 \\
\hline Lymphocyte, \% & 15.1 & 11.6 & 18.7 & 25 \\
\hline Platelet, $10^{3} / \mu \mathrm{L}$ & 127 & 122 & 155 & 182 \\
\hline C-reactive protein, $\mathrm{mg} / \mathrm{dL}$ & 8.15 & 9.01 & 5.05 & \\
\hline Erythrocyte sedimentation rate, $\mathrm{mm} / \mathrm{hr}$ & & 55 & 68 & 74 \\
\hline Ferritin, ng/mL & & 1698 & 1723 & 1880 \\
\hline D-dimer, $\mu \mathrm{g} / \mathrm{mL}$ & 0.70 & 1.11 & & \\
\hline LDH, U/L & 321 & 321 & 316 & \\
\hline Albumin, g/dL & 4.1 & 4.1 & 3.6 & 3.8 \\
\hline Prothrombin time, s & 14.0 & & & \\
\hline Aspartate aminotransferase, U/L & 38 & 33 & 31 & 42 \\
\hline Alanine aminotransferase, U/L & 31 & 30 & 32 & 53 \\
\hline Creatine kinase, U/L & 46 & 50 & 51 & 49 \\
\hline Interluekin-6, pg/mL & 55.6 & & & \\
\hline High-sensitivity troponin, ng/L & 6.4 & 6.8 & 6.1 & \\
\hline
\end{tabular}

WBC, white blood cells; LDH, lactate dehydrogenase.

drawn at admission and resulted 3 days later at 55.6. A procalcitonin level was not drawn. A repeat COVID-19 test was drawn and resulted positive the next day. His initial COVID-19 test drawn in "COVID clinic" also returned positive.

A portable chest radiograph revealed a nonspecific patchy airspace opacity within the right lower lobe. A computed tomography pulmonary angiography (Figure 1) revealed multi-focal air-space opacities, which can be seen in multi-focal community-acquired pneumonia. Further discussion with radiology revealed that multi-focal peripheral ground-glass opacities could be seen, which are commonly reported features of COVID-19. His electrocardiogram findings revealed a regular rate and rhythm with no ST changes or $\mathrm{T}$-wave inversions.

The patient was admitted to the hospital due to his ambulatory desaturation, report of chest tightness, duration of illness, and his universally severely elevated inflammatory markers. He was placed on ceftriaxone $1 \mathrm{~g}$ once daily and azithromycin $500 \mathrm{mg}$ once daily. He was placed in a negative pressure room with airborne isolation precautions. He was maintained on continuous pulse oximetry and telemetry. He remained on room-air during his 3-day hospital course and experienced no episodes of dyspnea. His chest tightness resolved. His lymphopenia and thrombocytopenia improved, while his inflammatory markers continued to elevate (Table 1). His total white blood cell count, creatine kinase, and high-sensitivity troponins all remained within normal limits. His appetite improved on day 3 of admission, and the patient began to feel more energy. His ambulatory oxygen saturation dropped to $87 \%$ on ambulation but promptly returned to $96 \%$. Given his significant clinical improvement, the patient was discharged. He was given home isolation recommendations per the US Centers for Disease Control and Prevention guidance and was told to contact the local public health department to receive additional instruction. A telephone follow-up 2 days post-discharge revealed significant clinical improvement. A follow-up phone call 4 days post-discharge revealed the patient had achieved complete symptom resolution.

\section{Discussion}

We reviewed the literature for clinical and laboratory predictors of poor outcomes in COVID-19. We identified 5 retrospective studies and 1 prospective cohort study (Table 2, Table 3). Four COVID19 comparison groups were identified: severe versus non-severe disease, non-survivor versus survivor, 
Table 2. Characteristics of Included Studies

\begin{tabular}{|c|c|c|c|c|}
\hline Study & Nation of Origin & COVID-19 Comparison Groups & Study Design & Sample Size N \\
\hline Mo et al. ${ }^{4}$ & China & Refractory vs Non-refractory & Retrospective cohort & 155 \\
\hline Wu et al. ${ }^{6}$ & China & ARDS vs No ARDS & Retrospective cohort & 201 \\
\hline Zhou et al. ${ }^{7}$ & China & Non-survivor vs Survivor & Retrospective cohort & 191 \\
\hline Zhang et al. ${ }^{8}$ & China & Severe vs Non-severe COVID-19 & Retrospective cohort & 140 \\
\hline Du et al. ${ }^{9}$ & China & Non-survivor vs Survivor & Prospective cohort & 179 \\
\hline Li et al. ${ }^{10}$ & China & Non-survivor vs Survivor & Retrospective cohort & 102 \\
\hline
\end{tabular}

ARDS, acute respiratory distress syndrome; vs, versus.

refractory versus nonrefractory COVID-19, and acute respiratory distress syndrome (ARDS) versus no ARDS groups. We will categorize the severe, non-survivor, refractory, and ARDS groups into a poor outcomes description in our discussion below. Of note, this review will not evaluate predictors of myocarditis in COVID-19, a known cause of disease mortality.

\section{Clinical Course}

Fever, cough, and fatigue are the most commonly reported symptoms of COVID-19. ${ }^{3-6}$ A recent COVID-19 meta-analysis substantiated this by reporting the most common disease-related symptoms as fever (82\%; 95\% confidence interval [CI]; $56 \%$ to $99 \% ; n=4410)$, cough $(61 \%$; $95 \%$ CI, $39 \%$ to $81 \%$; $\mathrm{n}=3985)$, muscle aches and/or fatigue $(36 \% ; 95 \%$ CI, $18 \%$ to $55 \%$; $\mathrm{n}=3778)$, and dyspnea $(26 \%$; $95 \% \mathrm{CI}$, $12 \%$ to $41 \% ; n=3700) .{ }^{11}$ The duration of symptoms is also an important part of the clinical evaluation. There are 2 reported peaks of COVID-19. The first occurs at 14 days and the second occurs at 22 days. ${ }^{12}$ One retrospective study reported median days to admission of $8 .^{8} \mathrm{~A}$ second retrospective study reported median days to intubation of 14.5 . $^{7}$ Two additional retrospective studies reported mean days to death of 11 and 17.8 days. $^{5,13}$

\section{Co-Morbidities}

Older age and comorbidities are well-accepted risk factors for poor outcomes. A meta-analysis revealed over twice the odds of developing severe disease for patients with hypertension (odds ratio $[\mathrm{OR}]=2.36$; 95\% CI 1.46-3.83), cardiovascular disease (OR = 3.42 ; $95 \%$ CI, 1.88-6.22), and respiratory disease $(\mathrm{OR}=2.46$; 95\% CI, 1.76-3.44). However, high statistical heterogeneity was acknowledged. ${ }^{14}$ Additional retrospective studies comparing the prevalence of comorbidities in COVID-19 cases revealed similar, but inconsistent findings (Table 3).

\section{Laboratory Markers}

COVID-19 patients commonly have lymphopenia. ${ }^{4,8,15}$ One study determined that lymphopenia could predict disease severity. From day 10 to 12, a lymphocyte percentage of less than $20 \%$ predicted "pre-severe" disease, while a percentage $>20 \%$ predicted "moderate" disease that is curable. From day 17 to 19 , a lymphocyte percentage $<5 \%$ predicted severe illness requiring intensive care level care, while a percentage $>20 \%$ was more reassuring. ${ }^{16}$ It is worth noting that lymphopenia can also be seen in autoimmune conditions, stress, acute infection, recent surgery, immunosuppressive drugs, and even as incidental findings in the elderly. ${ }^{17}$ Leukocytosis is not consistently present but is associated with poor outcomes. This is likely due to sepsis, a reported complication of COVID-19. Inflammatory markers are commonly elevated (Table 3). The erythrocyte sedimentation rate is nonspecific. Elevated lactate dehydrogenase, C-reactive protein, prothrombin time, and $\mathrm{D}$-dimer are more commonly cited as predictors of severe disease. ${ }^{6,7,9,10,15,18}$ Two retrospective studies cited 245 and 450 as cutoff values, respectively, for lactate dehydrogenase. ${ }^{6,7}$ One retrospective study cited 5 as the cutoff value for C-reactive protein. ${ }^{6}$ While ferritin is not consistently elevated, 2 retrospective studies cited 300 as a cutoff for predicting poor outcomes. 6,7

\section{Conclusion}

The 2020 COVID-19 pandemic introduced a novel conundrum for the medical community. Hospital admission risks must be weighed against the benefit of intervening at the earliest sign of complications. A firm understanding of clinical and laboratory indices 


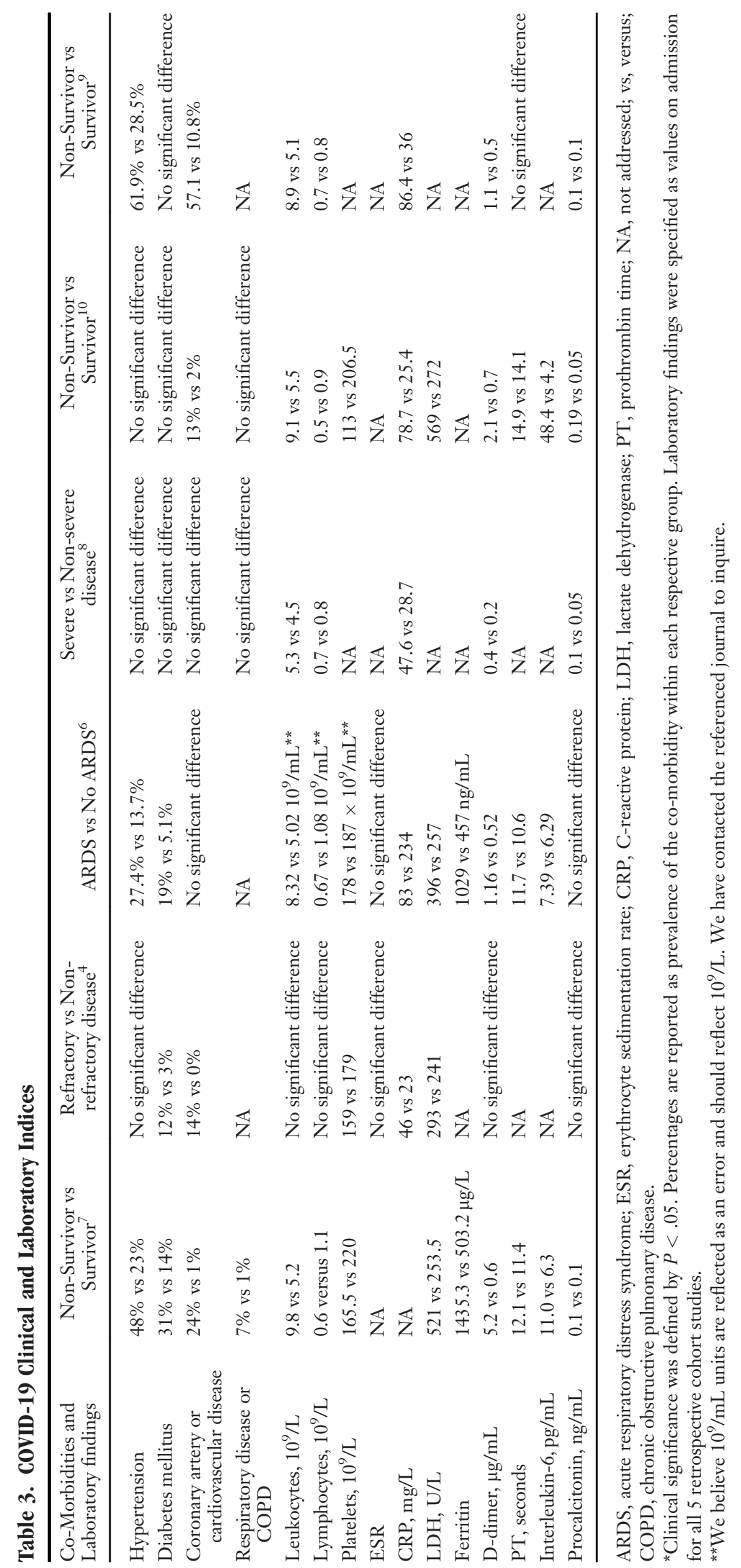


are a crucial part of this decision. We propose 3 basic steps to this decision: (1) consider comorbidities, specifically hypertension, diabetes mellitus, cardiovascular disease, and respiratory disease, (2) consider the duration of illness, with 2 peaks of illness occurring at 14 and 22 days, and (3) evaluate laboratory indices with a focus on lymphopenia, lactate dehydrogenase $>245$, C-reactive protein $>5.0$, ferritin $>300$, and an elevated $\mathrm{D}$-dimer. As an additional point, our case report and literature review demonstrate that while COVID-19 can cause significantly elevated inflammatory markers, it remains important to interpret these laboratory findings within the context of a comprehensive clinical evaluation. ${ }^{19}$ Future studies covering interleukin-6, procalcitonin, and other lab markers may provide additional value. ${ }^{20,21}$

Limitations of this review include a lack of studies completed in the United States. Population and clinical practice habits may vary between the United States and China. Additional studies evaluating local populations would help provide additional clarity. Relatively small sample sizes, a wide range of lab results, and statistical heterogeneity detracts from the statistical power. Lastly, 5 of the 6 evaluated studies were performed retrospectively.

The authors thank Dr. Paul Clark for his consultation and review of the patient's chest CT scan findings.

To see this article online, please go to: http://jabfm.org/content/ 34/Supplement/S186.full.

\section{References}

1. Wu Z, McGoogan J. Characteristics of and important lessons from the Coronavirus disease 2019 (COVID19) outbreak in China. summary of a report of 72314 cases from the Chinese Center for Disease Control and Prevention. JAMA 2020;323:1239.

2. Lake M. What we know so far: COVID-19 current clinical knowledge and research. Clin Med (Lond) 2020;20:124-7.

3. Singhal T. A review of Coronavirus disease-2019. Indian J Pediatr 2020;87:281-6.

4. Mo P, Xing Y, Xiao Y, et al. Clinical characteristics of refractory COVID-19 pneumonia in Wuhan, China. Clin Infect Dis 2020 Mar 16, ciaa270.

5. Leung C. Clinical features of deaths in the novel coronavirus epidemic in China. Rev Med Virol 2020;30:e2103.

6. Wu C, Chen X, Cai Y, et al. Risk factors associated with acute respiratory distress syndrome and death in patients with Coronavirus disease 2019 pneumonia in Wuhan. JAMA Intern Med 2020;180:934-43.

7. Zhou F, Yu T, Du R, et al. Clinical course and risk factors for mortality of adult inpatients with COVID-19 in Wuhan, China: a retrospective cohort study. Lancet 2020;395:1054-62.

8. Zhang J, Dong X, Cao Y-Y, et al. Clinical characteristics of 140 patients infected with SARS-CoV-2 in Wuhan, China. Allergy 2020;75:1730-41.

9. Du R-H, Liang L-R, Yang C-Q, et al. Predictors of mortality for patients with COVID-19 pneumonia caused by SARS-CoV-2: a prospective cohort study. Eur Respir J 2020;5 5:2000524.

10. Li K, Chen D, Chen S, et al. Predictors of fatality including radiographic findings in adults with COVID-19. Respir Res 2020;21:146.

11. Nascimento I, Cacic N, Abdulazeem HA, et al. Novel Coronavirus infection (COVID-19) in humans: a scoping review and meta-analysis. J Clin Med 2020;9:941.

12. Ruan Q, Yang K, Wang W, et al. Clinical predictors of mortality due to COVID-19 based on an analysis of data of 150 patients from Wuhan, China. Intensive Care Med 2020;46:846-8.

13. Ruan S. Likelihood of survival of coronavirus disease 2019. Lancet Infect Dis 2020;20:630-1.

14. Yang J, Zheng Y, Gou X, et al. Prevalence of co-morbidities in the novel Wuhan coronavirus (COVID19) infection: a systematic review and meta-analysis. Int J Infect Dis 2020;94:91-5.

15. Yan L, Zhang H-T, Gonclaves J, et al. An interpretable mortality prediction model for COVID-19 patients. Nature Machine Intelligence 2020;2:283-8.

16. Tan L, Wang Q, Zhang D, et al. Lymphopenia predicts disease severity of COVID-19: a descriptive and predictive study. Sig Transduct Target Ther 2020; $5: 33$.

17. Brass D, McKay P, Scott F. Investigating an incidental finding of lymphopenia. BMJ 2014;348: g1721.

18. Poggiali E, Zaino D, Immovilli P, et al. Lactate dehydrogenase and C-reactive protein as predictors of respiratory failure in CoVID-19 patients. Clin Chim Acta 2020;509:135-8.

19. Sarzi-Puttini P, Giorgi V, Sirotti S, et al. COVID19, cytokines and immunosuppression: what can we learn from severe acute respiratory syndrome? Clin Exp Rheumatol 2020;38:337-42.

20. Galli C, Plebani M. Clinical laboratory and SARSCoV-2 infection: where do we stand? Clin Chem Lab Med 2020;58:1139-41.

21. Lippi G, Plebani M. Procalcitonin in patients with severe coronavirus disease 2019 (COVID-19): a meta-analysis. Clin Chim Acta 2020;505:190-1. 